\title{
Improving Performance in Supply Chain
}

\author{
Camelia Ioana Ucenic $^{1, *}$, Claudiu Ratiu ${ }^{1}$ \\ ${ }^{1}$ Technical University Cluj Napoca, Faculty of Machine Building, Romania
}

\begin{abstract}
The performance has different meanings and ways of defining and measurement. One cannot evaluate the supply chain of a company without using the concept of performance. There are two ways of measuring it: with qualitative and with quantitative instruments. The quantitative measures include financial and non-financial appraise. This article analysis some of the most used metrics. Resources, flexibility and output are listed among them. The non-financial performance measurements will be analysed from the point of view of the company as well as from the point of view of several of its main customers. The customer evaluation will be used in the second stage for describing the qualitative performance of the analysed supply chain. Customer satisfaction score, customer effort score and net promoter score will be calculated as framework for the qualitative performance of supply chain. The results will provide managerial information for the improvement of this supply chain.
\end{abstract}

\section{Introduction}

It is very difficult to provide a general framework for describing and measuring the performance of a supply chain. The industrial and non-industrial systems developed a wide variety of supply chains. Their structure was a linear one at the beginning but in time it was changed. Nowadays can be noticed very often a network type structure. Quantitative [1] and qualitative [2] measures can be used for supply chain performance. Problem identification is the first step of the process. In the second stage, it is necessary to describe the domain of measurement as well as the selected indicators and the relationship among them [3].

The literature review mentions a great amount of indicators from different fields of approaches. There is very complicated to select the most appropriate among them. It is necessary to introduce a set of measurement and selection assumptions in order to increase the quality of analysis. If the set of indicators is too large appears the danger of information dilution. A small number of indicators decreases the quality of performance measurement. The reports resulted after performance analysis are important tools for decision making in the process of planning, monitoring and controlling. The managers can make decisions at all managerial levels and at different time horizons.

There are many studies at the level of supply chain performance and different rankings. For example, Gartner Supply Chain Top 25 ranks every year the companies from specific fields of activity according with their supply chain performance. It makes use of financial and

\footnotetext{
* Corresponding author: cameliaucenic@gmail.com
} 
opinion indicators. An overall weighting of 50-50 was used for the final score. The financial indicators had different weight in the formula: return of assets ROA 25\% (net income / total assets), inventory turns $15 \%$ (cost of sold goods / inventory) and revenue growth $10 \%$ [4].

\section{Defining the supply chain performance}

The supply chain performance is described as the capacity of the supply chain to deliver with minimum logistic cost the right product at the correct time and in the correct specified location [5]. The delivered products must be in the specified quantities from customers' orders and must have the required quality. The final customer has to receive the products and services at the minimum cost [6].

Other authors define supply chain performance as the overall performance of the processes which are included in the companies' supply chain [7]. Each product has its own unique supply chain. The upstream and downstream relationships between its activities and processes provide the design of the supply chain. The changes in the components of a supply chain will impact its performance level. A correct approach has to define the performance measures as well as the performance measurement systems.

Supply chain performance assessment implies the following aspects:

- defining the indicators: they have to be clear expressed and to allow to be applied for each component of the supply chain,

- perspective of indicators: different components of supply chain may have different approaches regarding an indicator; all parts should agree and decide which is the selected perspective,

- the way of data capturing: the same way of capturing data has to be used in all supply chain components,

- confidentiality: different companies from the same supply chain do not accept to reveal all internal information to the third parties [8].

\section{Type of metrics for supply chain performance}

Nowadays one of the main challenges when one evaluates the supply chain performance is to decide which the performance metrics are. It has to be specified how are calculated these metrics and how data is obtained. The performance metrics have the following functions: control, improvement and communication [9]. The control function allows to the participants from supply chain to quantity and control the performance of supplies. If the performance level was not reached, the gap between the results and the selected targets can be improved. All stakeholders must know the performance metrics.

The measurement of supply chain performance using a single metric is not recommended. Some authors proposed the usage of three main categories of performance measures: resources, output and flexibility [10]. The objective of performance measure type resources is a high level of efficiency, the goal of output performance measures is a high level of customer service which helps the companies to avoid the transition of customers to other supply chains. The aim of flexibility performance measures is a great ability to answer fast to the demands of a changing environment.

Table 1. Resources, output and flexibility indicators.

\begin{tabular}{|c|c|c|}
\hline Resources & Output & Flexibility \\
\hline Manufacturing cost & Number of produced units & Increased customer satisfaction \\
\hline Distribution cost & $\begin{array}{c}\text { Number of on-time } \\
\text { delivery }\end{array}$ & Reduction of late orders \\
\hline
\end{tabular}




\begin{tabular}{|c|c|c|}
\hline Inventory cost & Product quality & Flexibility to demand variation \\
\hline $\begin{array}{c}\text { Return on } \\
\text { investment }\end{array}$ & Customer satisfaction & $\begin{array}{c}\text { Flexibility to adapt to new market } \\
\text { requirements }\end{array}$ \\
\hline Total cost & Sales, profit & $\begin{array}{c}\text { Range flexibility } \\
\text { (to what extend can change an operation) }\end{array}$ \\
\hline
\end{tabular}

Another very common approach of the supply chain performance indicators is the one from financial point of view. It divides the indicators in financial and non-financial. Some of the indicators and their association with the managerial levels are presented in the table 2.

Table 2. Financial and non-financial indicators [11].

\begin{tabular}{|c|c|c|}
\hline Financial & Non-financial & Level \\
\hline Rate of return on investment & Delivery lead time & \multirow[t]{4}{*}{ Strategic } \\
\hline Delivery performance & Supplier lead time against industry norm & \\
\hline Variation against budget & Flexibility of service for customer needs & \\
\hline Net profit vs. productivity ratio & Customer perceived product value & \\
\hline Delivery reliability & Accuracy of forecast techniques & \multirow[t]{4}{*}{ Tactical } \\
\hline Supplier cost saving & Purchase order cycle time & \\
\hline & Order entry methods & \\
\hline & Product development cycle time & \\
\hline Cost per operation hour & Capacity utilisation & \multirow[t]{4}{*}{ Operational } \\
\hline Information carrying cost & Supplier rejection rate & \\
\hline Work in progress inventory & Frequency of delivery & \\
\hline Scrap level inventory & Quality of delivery documentation & \\
\hline
\end{tabular}

\section{Case study}

This article uses the non-financial indices in order to evaluate the performance of the supply chain. The evaluation was done for the supply chain of an automotive company located in Transylvania. The name of the company as well as the name of its customers were not revealed due to confidentiality reasons.

There are many non-financial indicators as was previously described. This study uses the customer satisfaction indicator as measurement for the supply chain performance. Customer satisfaction indicator was selected because a great percentage (89\% according with 2011 Customer Experience Impact Report) of customers stop doing business with a firm after they experienced a low customer service. The loss of a customer generates an increase of costs because it is more expensive to attract a new one than retaining the existing ones. The customer satisfaction can be also measured and evaluated through different ways. The customer satisfaction score, customer effort score and net promoter score were chosen for the assessment.

Data collection took place in the interval January - May 2017. The methodology used qualitative and quantitative designs. A structured interview was conducted having as starting point the customer data basis selection given by the firm. The selection considered the main customers from the point of view of their impact in the firm's supply chain. The focus was on the persons who have expert knowledge at supply chain level. The total number of answer was 38 . The selection step was 3 . Primary and secondary data sources were used in the final evaluation of supply chain performance. 


\subsection{Customer satisfaction score CSS}

The customer satisfaction score is very often used by the companies in their tries to measure and improve firm performance. Different persons rate their satisfaction in different manners in accordance with their culture. Some cultures tend to have a more simple scale to estimate the satisfaction but other have more satisfaction levels. This is the reason which generates different scale ranges for customer satisfaction score. The scale can be: 1-3, 1-5 or 1-10. A large range is not always a better one. For example, the US government employed a 4 star rating [12].

The general way to measure the customer satisfaction score is to address the following question: "How would you rate your global satisfaction of the service you received?" The customer has to rate his degree of satisfaction on the scale 1 to $5: 1=$ very unsatisfied, $2=$ unsatisfied, $3=$ neutral, $4=$ satisfied and $5=$ very satisfied. The composite customer satisfaction score CCSS is calculated as the average result.

This study calculates the CSS on a scale of $0-100 \%$, where the 100 illustrate the total customer satisfaction. Only the number of respondents which rated their satisfaction with score 4 and score 5 are included.

$$
S S C=\frac{\text { Number of satisfied customers }}{\text { Number of satisfaction survey responses }} \times 100
$$

The literature review shows that this is a correct approach because only the customers with the two highest possible response rating correctly predict the customer retention [13].

The following questions were included in the survey:

- Q1: How would you rate your global satisfaction regarding our quality of the product P1? $\mathrm{P} 1$ is the product with the greatest impact in the volume of sales

$$
S S C_{1}=\frac{24}{38} \times 100=63,15 \%
$$

- Q2: How would you rate your global satisfaction regarding our delivery time for the product P1? ( answer shows responsiveness in supply chain)

$$
S S C_{2}=\frac{17}{38} \times 100=44,73 \%
$$

- Q3: How would you rate your global satisfaction regarding our product variety?

$$
S S C_{3}=\frac{31}{38} \times 100=81,57 \%
$$

- Q4: How would you rate your global satisfaction regarding our ability to answer to your changing needs? ( answer shows flexibility in supply chain)

$$
S S C_{4}=\frac{11}{38} \times 100=28,94 \%
$$

The results show that the product variety obtained the highest customer satisfaction score. The worst aspect in the supply chain performance is its lack of flexibility, followed by a bad score regarding the responsivity. The SSC for quality of products has the second higher result but it is less than $80 \%$, so still need special attention.

\subsection{Customer effort score CES}

The customer effort score is important when appear issues which have to be solved. The customer effort score is among the newest performance indicators. It was introduced by CEB (Corporate Executive Board) in 2010. CEB is a global best practice insights and technology company. "CES is $1.8 \mathrm{x}$ more predictive of customer loyalty than customer satisfaction (CSAT) measures, plus it is two times more predictive than Net Promoter Score (NPS)" [14]. 
The problem resolution implies a certain level of effort from the customer point of view. The customer effort score is important because it was demonstrated that customers tend to punish a bad service more often than to recompensed a good service. The clients appreciate more if their problem resolution is as easy as possible. A customer is loyal when his experience was effortless. It is recommended to address the CES question as soon as possible after the customer concluded his experience of interest.

The CES is calculated by asking an answer at the following question: "How easy made for me the company to handle the problem X?" The answer is rated on a scale from 1 to 7 , where 1 means very low effort and 7 means a very high effort. The scale for CES: $1=$ strongly disagree, $2=$ disagree, $3=$ somewhat disagree, $4=$ neutral, $5=$ somewhat agree, $6=$ agree, $7=$ strongly agree. The scale can be reduced $1-5$ or $1-3$ but the results loose part of significance.

The scale $1-3$ was used. At the first tries with a 7 steps scale, the respondents couldn't decide fast a proper answer. The scale: $1=$ difficult, $2=$ neutral, $3=$ easy.

$$
\text { CES }=\% \text { Easy }-\% \text { Difficult }
$$

This way of calculation was used because it is done in the same way by the majority of leading American brands. Action plan implementation is easier in this approach instead of calculation CES with the ratio total sum of scores / number of scores.

The following questions were included in the survey:

- Q1: How easy was to make an order using the website?

$$
C E S_{1}=42 \%-15 \%=27 \%
$$

- Q2: How easy was to solve your complain in terms of time?

$$
C E S_{2}=37 \%-26 \%=11 \%
$$

It is observed a better CES from the point of view of making orders. The long time for solving complains affect the performance of supply chain.

\subsection{Net promoter score NPS}

The net promoter score is the most common method to evaluate the customer loyalty. NPS together with CES allow to the company to compare the results and to provide a better action plan. The question is if the customer will recommend you to somebody else or not. The scale starts at 0 and goes until 10. The significance of each level is presented in the next table.

Table 3. Net promoter score scale [12].

\begin{tabular}{|c|c|c|c|c|c|c|c|c|c|c|c|}
\hline Code & 0 & 1 & 2 & 3 & 4 & 5 & 6 & 7 & 8 & 9 & 10 \\
\hline Meaning & Not a chance & & & & & Neutral & & & & & Very likely \\
\hline Type & \multicolumn{1}{|c|}{ Detractors } \\
\hline
\end{tabular}

The detractors have a score from 0 to 6 . They are unsatisfied customers that complain about company, products and services and affect the image and reject other potential customers. The passive customers have score 7 or 8 . They are satisfied but don't have loyalty and can be strongly influenced by the competition. They don't damage company image but also don't recommend it to other customers. The promoters have the score 9 and 10. They are happy, satisfied by the company. They keep on buying its products and more than this will recommend it to other people.

$$
\begin{array}{r}
\text { NPS }=\% \text { Promoters }-\% \text { Detractors } \\
N P S=32 \%-18 \%=14 \%
\end{array}
$$


A NPS of $14 \%$ is not sufficient. It illustrate that many customers are passives. The company performance can be increased by pushing them from "passive" category to "promoters" category. NPS together with other non-financial and financial indicators can help the decision makers in their process to increase the supply chain performance.

\section{Conclusions and recommendations}

The main objective of this study was to measure and evaluate the supply chain performance of a company from automotive sector using opinion non-financial performance indicators. The company must increase its supply chain performance. This can be done starting from the lowest scores. It must track the negative ratings. Another recommendation is to decrease the time for responding to the customers. The literature review suggest an answer in less than 10 hours for generating scores above $90 \%$.

The percent of resolved issues has to increase. This has to be done in a single interaction, as much as possible. The implemented changes have to be communicated to the clients. The client will feel he is valued for the given input. Another urgent requirement is to reduce the time for complain solving. Customer effort score can be calculated using a scale from 1 to 7 , but it is necessary to address the question to persons with more experience in supply chain aspects and with higher positions in the organisational structure.

CES and NPS provide to service leaders a holistic company image: CES gives the service perspective, while NPS gives answers from business perspective. The firm has to decide in the final action plan if wants to reduce the customer effort or to increase the promoters.

Further research can be done. The number of non-financial indicators can be increased and obtain a better evaluation framework. They can be analysed in parallel with financial indicators. A larger set of measures can be proposed in order to increase the performance of supply chain from this company.

\section{References}

1. C. Sheperd, H. Gunter, Behavioral Operations in planning and scheduling, J.C. Francsoo (Eds), Springer Verlag (2016)

2. F.T.S. Chan et all, Management Decision 41 (7), 635-642 (2003)

3. D. Leonczuk, J. Business Management and Education 14 (1), 103-105 (2016)

4. $* * *$ https://www.gartner.com/doc/2493115/gartner-supply-chain-top-\#1739401341

5. H. Zhang, S.C. Okoroafo, EMR 4 (1), 38-48 (2015)

6. G.D. Whitten, K.W. Green, P.J. Zelbst, Int J of Operations \& Production Management 32 (1), 28-48 (2012)

7. M. Srinivasan, D. Mukherjee, A. Gaur, European Mg. J 29, 260-271 (2011)

8. H. Stadtler, C. Kilger, Supply chain management and advanced planning. Concepts, Models, Software and Case studies. 4th ed., Springer-Verlag, Germany (2008)

9. S.A. Melnyk, D.M. Stewart, M. Swink, JOM 22(3), 209-217 (2004)

10. B.M. Beamon, Int. J. of Operations \& Production Management, 19 (3), 275-292 (1999)

11. B. Gunasekaran, C. Patel, R.E. McGaughey, Int. J of Production Economics 87(3), 333-347 (2004)

12. *** https://www.userlike.com/en/blog/6-proven-methods-for-measuring-yourcustomer-satisfaction

13. *** https://blog.emolytics.com/customer-experience/customer-satisfaction-score-csat-kpi/

14. M.Dixon, K.Freeman, N.Toman, Harvard Business Review, (2010) 